\title{
Itinerario y desafíos de la formación inicial docente en Chile ${ }^{*}$
}

\author{
Héctor Gómez Cuevas
}

\begin{abstract}
Resumen
El presente espacio reflexivo, aborda las tensiones y desafíos que emergen del itinerario de programas y políticas en torno a la Formación Inicial Docente en Chile (FID) en la última década. En este sentido, se evidencia la influencia de los cambios globales en este ámbito y la incidencia en las rutas de innovación y cambio llevadas a cabo en América Latina y en Chile. Las características de estos nuevos escenarios muestran una preocupación por la excesiva desregulación del sistema de educación superior en el ámbito de la formación docente, sin embargo, las acciones realizadas y los lineamientos a futuro, desafían a las instituciones formadoras a cambiar en un contexto de extremo control del diseño curricular.
\end{abstract}

Palabras clave: Formación Inicial Docente, Programas y Políticas Públicas, Diseño Curricular

\section{Itinerary and challenges in initial teacher training in Chile}

\section{ABSTRACT}

This reflection addresses strains and challenges emerging from the itinerary of programs and policies related to the initial teacher training in Chile in the last decade. In this sense, it notes the influence of global changes in this area and the impacts on pathways for innovation and change in Latin America and Chile. The characteristics of these new scenarios point to a concern for the excessive deregulation of the higher education system in the field of teaching training. However, actions taken and future guidelines challenge training institutions to change in a context of extreme control of curriculum design.

Key words: Initial teacher training, programs and policies, curriculum design

* Este artículo forma parte de los resultados de la tesis de magíster de la Pontificia Universidad Católica de Chile titulada "ANÁLISIS CRÍTICO DEL DISCURSO DEL CURRÍCULUM Y DE GÉNERO DE FORMACIÓN INICIAL DOCENTE DE PEDAGOGÍA EN EDUCACIÓN BÁSICA EN CHILE, 2013.

* Magíster en Ciencias de la Educación. Mención Currículum. Pontificia Universidad Católica de Chile. Académico Escuela de Educación Básica de la Facultad de Educación, Universidad Católica Silva Henríquez. 
Una serie de investigaciones y estudios (IIPE-UNESCO, 200I; Vaillant, 2004; Latorre, 2005), han señalado el bajo impacto de la formación inicial docente ${ }^{\mathrm{I}}$ entregada por las universidades formadoras, en tanto señalan que los/as maestros/as al iniciarse profesionalmente en este ámbito, tienden a refugiarse en imágenes previas de experiencias como alumnos/as, o terminan siendo sometidos a las demandas del contexto escolar, (des) aprendiendo o (re) aprendiendo experiencialmente a ser profesores/as a través de sus propias prácticas y de otros/as colegas.

Este diagnóstico instala el primer desafio a un campo teórico-práctico cuyos límites tienden a ser complejos e indeterminados, ante lo que se hace necesario clarificar desde dónde se refiere la FID. Al respecto, Ruth Mercado (2010), en referencia a Schön (1992), señala que la formación de maestros/as debe ser entendida como un proceso continuo, que no debe parcelarse en etapas iniciales, prácticas y posteriores, por lo cual es relevante hablar de procesos permanentes y continuos.

Asimismo Perrenoud (200I) señala que la FID, más que un concepto neutro, debe ser entendido como concepto socialmente construido, que varía en el marco de las relaciones con las condiciones sociales e históricas, requiriendo como componentes básicos una formación humana integral, una sólida preparación en disciplinas y competencias relacionadas con el desarrollo del aprendizaje y una introducción a la práctica de la enseñanza, bajo la guía de un/a maestro/a experimentado/a.

A su vez, Vaillant (2004) complementa lo anterior, destacando que la FID representa "uno de los problemas más difíciles enfrentados por los gobiernos y las sociedades por sus implicancias políticas, ideológicas y financieras" (2004: 6).

Lo señalado anteriormente demuestra con claridad, la fragilidad y complejidad del tratamiento de la formación de profesores/as, en cuanto a la participación de múltiples discursos en juego, sobre todo respecto a los límites de la formación, la diversidad de contextos

I En adelante FID. 
en donde se desarrolla y el nivel de responsabilidad asignado a este ámbito en el contexto global de la educación y calidad educativa.

En este contexto, el presente artículo expone el conjunto de desafíos que ha enfrentado el campo de la FID a nivel internacional, con énfasis en el itinerario de programas y políticas en este ámbito, tomando como punto de partida la finalización del Programa de Fortalecimiento de la Formación Inicial Docente (Proyecto FFID), en tanto forma parte de la política más significativa en este ámbito tras el retorno al sistema democrático en Chile. Como punto cúlmine de este itinerario, se considera la elaboración y publicación del documento de Estándares Orientadores para Egresados de carreras de Educación Parvularia, Básica y Media entre 20I I y 2012.

Luego de dar cuenta del itinerario, el artículo se propone describir los desafíos generados en el contexto actual, caracterizado por un panorama de políticas y programas ministeriales como INICIA, Beca Vocación de Profesor, los mismos Estándares, y el Proyecto de Ley de Carrera Profesional Docente, que desde sus propios discursos apuntan a la mejora de la 'calidad' de la FID, pero que a su vez generan complejos procesos de estandarización, homogeneidad, y control del diseño curricular que tensionan decididamente a la instituciones formadoras de docentes.

\section{Contexto Internacional}

A nivel nacional e internacional, la FID ha generado un ámbito de importancia crucial dentro del ámbito educativo, en tanto investigaciones como OECD (2004) "Teacher Matter" y el Informe Mackinsey (Barber y Mourshed, 2008), han puesto en el centro del debate sobre educación, la función de los/as docentes.

En dichas investigaciones se ha insistido en la necesidad de definiciones políticas en FID, que focalicen su atención en la atracción de jóvenes talentosos/as a la formación pedagógica, la existencia de una formación docente de calidad y la existencia de incentivos para retener a los/as mejores docentes en el aula (OECD, 2004; Barber\&Mourshed, 2008). 
Algunas experiencias internacionales al respecto muestran para el caso de Unión Europea, la adopción de dos modelos en la FID, uno que concibe la formación más extensa, en tanto el ingreso de los/ as estudiantes al sistema es a una edad más temprana, y un segundo más corta, por cuanto su ingreso es más tardío, tras haber recibido un título previo. En ambos modelos las calificaciones académicas representan el filtro más importante, condicionando a su vez la cantidad de vacantes en relación al mercado laboral y las políticas educativas. (Eurydice, 2002).

No obstante los aspectos comunes mencionados, existen diferencias sustantivas en Europa de acuerdo al énfasis curricular de las competencias profesionales o pedagógicas que asignan, y entre la FID y el ejercicio profesional, así como en las experiencias de prácticas profesionales. Sin perjuicio de ello, en la mayoría de los casos ha existido una intervención importante al currículum de formación en áreas como el uso de TIC, la atención de los/as inmigrantes y de las/os estudiantes con Necesidades Educativas Especiales, Transitorias o Permanentes, la gestión administrativa, la comunicación, el comportamiento en las instituciones educativas. (Latorre, 2005).

El caso de Estados Unidos, respecto de la FID ha considerado una serie de cambios sustantivos, como iniciativas gubernamentales. El primero de ellos ha significado la generación de estándares de calidad, así como de sistemas de medición, con el fin de influir en las condiciones formativas, laborales y salariales de los/as profesores/as. En segundo lugar, se ha generado una serie de cambios al currículum de formación, de modo "intercultural", de manera tal que se potencie al docente como un "profesional de la educación" capacitado/a para enfrentar una diversidad de situaciones en su práctica. Tercero, las instituciones formadoras han implementado certificaciones específicas en ámbitos de la formación, selección, continuidad y consistencia de la malla curricular, así como en los contenidos pedagógicos, disciplinares e interdisciplinares; y la carga horaria de las prácticas progresivas, vinculando de forma efectiva las instituciones formadoras con las escuelas. (Villegas-Reimers, 2002). 
A partir de estos antecedentes, es relevante destacar la importancia en Europa y Estados Unidos de la FID, quienes han ido progresivamente instalando estándares mínimos, evaluaciones del desempeño, procesos de selección al ingreso, prácticas progresivas, cambios curriculares, reformas políticas y una serie de reformas que apuntan a profesionalizar la labor docente, y en conjunto con ello elevar la calidad de la educación y los aprendizajes de los/as estudiantes.

En síntesis, a nivel internacional ha existido una cada vez mayor relevancia de la FID, considerando este ámbito como uno de los fundamentales en los cambios educativos, sobre todo por lo que plantea Manzi (2009), donde el mejoramiento en los logros de los sistemas educativos estaría fuertemente asociado a la calidad de los docentes. En este sentido, las políticas educativas implementadas en los diferentes contextos internacionales se enfocan hacia mejorar la calidad de la FID, mediante tres focos específicos: las mejoras en los currículos de formación, la atracción de mejores estudiantes a las carreras de pedagogía, mejorando el estatus profesional en términos simbólicos y materiales, y por último estableciendo políticas de estandarización de la FID, mediante la instalación de evaluaciones al egreso como formas de establecer estándares mínimos de desempeño en el ejercicio de la docencia.

\section{Contexto en América Latina}

En cuanto a América Latina, Vaillant (2004:17) señala que "la formación docente aparece como uno de los desafíos más críticos del desarrollo educativo; muchos maestros y profesores están muy mal preparados y además el cuerpo docente necesita un perfeccionamiento mediante un esfuerzo masivo de formación en servicio".

En la región, las experiencias han sido muchas, pero las variaciones entre ellas no han sido significativas, necesitándose a juicio de Vaillant (2009) un cambio de enfoque, en tanto la experiencia de las reformas en formación de profesores/as en la década de los 90 en América Latina, ha presentado un escaso impacto, y una serie de ellas no se reflejaron en las prácticas, las cuales siguieron obedeciendo a viejos modelos. 
En la actualidad, la región vive un proceso de absorción de las políticas internacionales en torno a la FID. Este proceso converge en la globalización de la cultura y la homogeneización de los discursos, que Cardelli y Duhalde (200I) sitúan como un proceso de transformaciones culturales derivadas de la mercantilización de los objetos de la cultura y en particular del conocimiento científico y técnico; de las acciones y discursos que dan nuevo impulso a la demanda educativa, generando una centralidad y uniformidad de la FID.

Los/as autores/as afirman además que las iniciativas en torno a la FID en la región, situadas en el marco de un modelo económico neoliberal, regulan las dimensiones de cultura de las instituciones de formación docente, el trabajo docente y el desarrollo curricular (Cardelli y Duhalde, 200I: 2), que en definitiva modelan un conjunto de prácticas que, a juicio de Torres (1996), desconocen los caminos anteriormente recorridos, las percepciones de los propios/as sujetos/ as, adoptan un enfoque vertical, las propuestas son homogéneas sin considerar la diversidad y las necesidades específicas, obvian la colectividad y se desvinculan con el contexto en donde se generan las reformas en torno a la FID.

En la recién pasada década, América Latina ha implementado una serie de estrategias político-educativas tendientes a "monitorear la continuidad de los contenidos pedagógicos e (inter)disciplinares; la innovación según avances científicos y tecnológicos; y la flexibilidad de las prácticas de enseñanza y procesos de aprendizaje, intentando atender a las emergentes transformaciones históricas y socioculturales de la región”. (Latorre, 2005: 72).

Los programas de FID a juicio de la autora, están insertos en las grandes reformas de "mejoramientos de la calidad con equidad" (Latorre, 2005), que han concertado intereses, necesidades y exigencias de los contextos internacionales y locales a fin de mejorar de forma sustantiva los índices educativos. 


\section{Itinerario de la Formación Inicial Docente en Chile (2002 - 2010)}

En el caso de Chile, se exponen un conjunto de antecedentes que han orientado la FID en general y de Pedagogía en Educación Básica en particular, considerando las medidas realizadas dentro de la Reforma Educativa de los 90, poniendo énfasis en el periodo 2002-20I I, que da cuenta de un conjunto de medidas para elevar la calidad de la FID, desde la evaluación y resultados del Programa de Fortalecimiento de la Formación Inicial Docente FFID (2002), hasta la publicación de Estándares Orientadores para Egresados de Carreras de Pedagogía en Educación Básica (201 Ia).

A modo introductorio, Ávalos (2008) enuncia que la FID en Chile representa "un sistema grande y fragmentado de carreras de pedagogía, carente de regulación externa y sobre el que existen dudas respecto a la capacidad docente de muchos de sus egresados" (276).

La trayectoria de la FID se debe contextualizar en el marco de un conjunto de iniciativas político-educativas de los diferentes gobiernos de Chile, quienes implementaron en el marco de las grandes reformas educativas de los $90-y$ con posterioridad-un conjunto de medidas, que apuntan hacia una transformación en las condiciones formativas, laborales y salariales del profesorado. Esta serie de medidas, a su vez, no pueden ser entendidas sino como respuesta a la baja calidad en los resultados obtenidos en las evaluaciones estandarizadas (SIMCE, PISA, TIMSS, PSU) por los/as estudiantes, y en el último periodo de los/as propios/as docentes en la Evaluación Docente e INICIA. (Mineduc, 2008, 2009, 2010).

Al respecto, Ávalos (2003) señala que en Chile, desde los años 90, se implementaron políticas públicas enfocadas en tres ámbitos específicos: mejorar condiciones de trabajo de los docentes, incentivo en el acceso a carreras de pedagogía entregando ayudas a los postulantes de altos puntajes en las pruebas de ingreso a la educación superior y en la formación docente.

A continuación se expone brevemente el itinerario de programas y políticas públicas en torno a la formación inicial docente en Chile. 


\section{Programa de Fortalecimiento de la Formación Inicial Docente (PFFID)}

El primero de ellos corresponde al Programa de Fortalecimiento de la Formación Inicial Docente (PFFID) desarrollado entre 1997 y 2002, el que consistía en un conjunto de recursos económicos destinados a mejorar la FID, a través de medidas específicas, tales como la incorporación de prácticas pedagógicas progresivas y el desarrollo de competencias en la escuela; definición de estándares de egreso para los/as estudiantes de pedagogía; actualización curricular; pasantías al extranjero de los/as formadores/as de docentes; mejoramiento de la infraestructura y el soporte tecnológico de las facultades de educación; y la atracción de los mejores puntajes académicos a las carreras pedagógicas (Ávalos, 2003, 2008 y MINEDUC, 2005).

Esta primera y gran iniciativa -por la cantidad de recursos y las instituciones participantes-, generó como resultados la renovación y ampliación de las prácticas profesionales, fomentó acciones para atraer a mejores egresados de la enseñanza media (becas), mejoras de las condiciones materiales asociadas a la formación docente (infraestructura, bibliotecas, recursos didácticos y equipamiento computacional), apoyó el mejoramiento de los equipos académicos y generó estándares de egreso para las facultades, que representaron la base para el Marco para la Buena Enseñanza. (Manzi 2009).

Cabe señalar que el proyecto FFID, si bien delineó una serie de políticas posteriores -sobre todo con la continuación de los proyectos MECESUP-, como programa propiamente tal fue descontinuado, presionados por demandas de matrícula para contribuir a su financiamiento (Manzi, 2009; Ávalos, 2009).

\section{Programa Mecesup}

El programa de Mecesup es financiado con recursos provenientes del Presupuesto de la Nación y de un crédito convenido con el Banco Internacional de Reconstrucción y Fomento (BIRF N ${ }^{\circ} 4404-$ $\mathrm{CH}$ ) y buscó promover las competencias necesarias que permitan aumentar la competitividad global, sostener el desarrollo económico 
y social y asegurar que ningún talento se pierda por diferencias en las oportunidades de aprendizaje.

Los objetivos generales del proyecto fueron: aumentar la efectividad del financiamiento público para la educación terciaria a través del incremento de la coherencia, equidad y calidad en el sistema, y por medio de un mejor desempeño, con mayor transparencia y rendición de cuentas públicas.

\section{Estándares de Desempeño para la Formación Inicial de Docentes (2000)}

La elaboración del documento de Estándares de Desempeño para la Formación Inicial de Docentes (2000), como parte del PFFID, significó un primer intento de establecer principios comunes a la formación de profesores/as en Chile, señalando que los estándares "son necesarios para que el Estado, como entidad responsable de la educación, tenga herramientas que le permitan garantizar que los maestros y las instituciones educadoras están desarrollando su función de manera adecuada” (MINEDUC, 2000: 4).

Esta declaración de principios configuró un intento concreto del gobierno de la época, para intervenir de forma directa la FID, estableciendo un marco conceptual acerca de la misión y tareas docentes, estándares de desempeño y su evaluación. En relación a estos estándares, se describieron una serie de criterios, indicadores y sugerencias que articularon en conjunto con documentos posteriores el marco regulatorio y técnico de la evaluación docente.

\section{Criterios de Acreditación de Programas de Pedagogía CNAP (2002)}

Durante 2002, la Comisión Nacional de Acreditación de Pregrado CNAP (actual CNA), determinó los Criterios para la Acreditación de las Carreras de Pedagogía (CNAP, 2002), (modificados nuevamente en 2007), que consideraba como insumo clave el documento de Estándares de Desempeño (2000). A partir de allí, un conjunto de programas de pedagogía, de forma voluntaria, y a partir de 2009 de 
forma obligatoria, fueron evaluados con disímiles resultados (Ley 20.I29 de Aseguramiento de la Calidad de la Educación Superior).

De este proceso de evaluación de los programas, a 2010 se señala que: el $96 \%$ está dentro del sistema de acreditación, lo que se traduce en 435 carreras de un total de 467; de estas 435, ocho resultaron no acreditadas, I3I acreditadas y 296 en proceso de acreditación. Respecto de los resultados, la mayoría de las carreras, 40 de las que se han presentado en proceso, han sido acreditadas por tres años, hay ocho carreras no acreditadas y sólo cuatro han llegado a los seis años de acreditación, ninguna a los siete. (CNA, 20I0).

\section{Asignación de Excelencia Pedagógica (2002)}

Durante el mismo año (2002) se llevó a cabo la implementación del Programa de acreditación para la Asignación de Excelencia Pedagógica (AEP), consistente en un programa del Ministerio de Educación cuyo objetivo es fortalecer la calidad de la educación a través del reconocimiento al mérito profesional de los docentes de aula.

Este reconocimiento consiste en una asignación que responde a lo dispuesto en la Ley I9.7I5 y el DFL N ${ }^{\circ}$ I de Educación, del I5 de enero de 2002, que busca evaluar el desempeño profesional de conocimientos, habilidades y competencias de excelencia, en docentes de aula de establecimientos del sector municipal o particular subvencionado. La obtención de la excelencia conlleva asociada una retribución económica, junto a la posibilidad de postular a la Red de Maestros de Maestros (desde 2004).

Cabe señalar que este sistema de acreditación de la excelencia se fundamentará en el documento Marco para la Buena Enseñanza (2003), a través de una prueba de conocimientos disciplinarios y pedagógicos, y de un portafolio, que contienen los distintos ámbitos del quehacer docente: conocimiento de las disciplinas, didáctica y contenido curricular, así como habilidades y competencias ejercidas por los docentes en el aula, todos ellos atendiendo el contexto de la educación chilena, su diversidad regional y sociocultural. 
De forma paralela al AEP, en 2003 se elaboró el documento Marco para la Buena Enseñanza (MBE), instrumento técnicopedagógico elaborado con expertos/as del Ministerio de Educación, de la Asociación Chilena de Municipalidades, y del Colegio de Profesores. Este documento, de suma vigencia en la actualidad, describe cuatro ámbitos de la buena enseñanza: la preparación de la enseñanza, la creación de un ambiente propicio para el aprendizaje de los/las estudiantes, la enseñanza para el aprendizaje de los/las estudiantes, y las responsabilidades profesionales. El documento marco fue elaborado con la intención de fortalecer a los/as docentes principiantes y experimentados/as, y a los programas y propuestas formativas, así como generar procesos de evaluación de la docencia en ejercicio. (MINEDUC, 2003).

\section{Informe sobre Educación Superior en Chile OCDE (2004)}

El año 2004 marca un punto de inflexión en la cronología de hitos que conforman la dinámica político-educativa de la FID, por cuanto la Organización para la Cooperación y el Desarrollo Económicos (OCDE) realiza un crítico informe sobre Educación Superior en Chile (OCDE, 2004), señalando que nuestro sistema adolece de: no poseer capacidad autorregulatoria, un crecimiento explosivo de la oferta universitaria, ausencia de estándares o criterios compartidos para fundamentar la formación docente, baja coherencia entre formación docente y requerimientos del sistema escolar, bajo status relativo de las Facultades de Educación al interior de las instituciones universitarias, escaso desarrollo de la investigación educacional y que la acreditación obligatoria no tendrá efectos inmediatos. (OCDE, 2004 - Manzi, 2009).

Cabe destacar el énfasis en las potentes debilidades en el ámbito educativo propiamente tal en las instituciones de educación superior. La cita a continuación grafica de forma clara el diagnóstico crítico que la OCDE realiza: El débil nexo entre las reformas y la formación inicial de profesores ayuda a crear una 'brecha de capacidad' mayor en la fuerza docente. Esto pone a la mayoría de los estudiantes del país en clases con profesores/as que, no por culpa propia, han sido preparados inadecuadamente para enseñar matemáticas, lenguaje 
y otras materias, al nivel requerido por el currículo chileno. Los considerables aumentos de sueldo a los profesores en los años 1990 han empezado a atraer alumnos de rendimiento mucho más alto a las facultades de educación. Pero el currículo de formación de profesores en las universidades no parece estar avanzando al mismo ritmo para proveer una preparación más fuerte en materias específicas o vincular los cursos de pedagogía al nuevo currículo. (OCDE, 2004: 9I).

\section{Evaluación Docente}

Durante el mismo año, se agrega un hito fundamental a la trayectoria de estrategias político-educativas en FID, en tanto se promulga la Ley I9.96I, sobre 'Evaluación Docente', la cual declara: que le corresponderá al Ministerio de Educación, a través del Centro de Perfeccionamiento, Experimentación e Investigaciones Pedagógicas (CPEIP), la coordinación técnica para la adecuada aplicación de los procesos de evaluación (Ley I9.96I, 2004). De esta forma, se da inicio a un sistema de evaluación del desempeño profesional tomando en consideración los dominios, criterios e instrumentos establecidos por el Ministerio de Educación, a través del CPEIP, y Comisiones Comunales de Evaluación Docente, quienes cumplirán la responsabilidad de aplicar localmente el sistema de evaluación, a cargo de evaluadores pares, es decir, profesores de aula que se desempeñen en el mismo nivel escolar, sector del currículo y modalidad del docente evaluado, aunque en distintos establecimientos educacionales que los docentes evaluados.

Evidentemente que los disímiles resultados tensionan a la FID, en tanto se asocian los niveles de logro a las carencias recibidas en su formación. Este y otros supuestos comienzan a ser parte del debate en torno a la formación de profesores/as, a lo que se suman los resultados de aprendizaje evaluados en el Sistema de Medición de la Calidad de la Educación (SIMCE). (Ávalos, 2009).

Luego de promulgada la primera inciativa de Ley sobre Evaluación Docente (19.96I, 2004), y el crítico informe sobre Educación Superior en Chile de OCDE (2004), se crea en 2005, por iniciativa del Ministerio de Educación, la "Comisión sobre Formación Inicial 
Docente”, la cual emitió un Informe Final que identificó importantes nudos problemáticos, tales como: la gestión institucional y el marco curricular que constatan la fragmentación en las líneas de formación, enfoques curriculares y perfiles de egreso, la escasa investigación educativa, y la desvinculación con el Ministerio de Educación, los centros escolares y las otras universidades, y en tercer lugar "se evidencia que las condiciones de (re)producción y distribución del conocimiento pedagógico e (inter)disciplinar "no se hacen cargo" de las operaciones representacionales y experiencias identitarias de sus actores protagónicos/as" (Comisión sobre Formación Inicial Docente, 2005).

\section{Docentes para un nuevo siglo (2005)}

Como parte de la serie 'Bicentenario', se desarrolla este informe que buscó como tarea central ofrecer un conjunto de reflexiones acerca de una educación de calidad para todos, ante lo cual señala que se requieren profesores/as bien formados/as que gocen de estimación social, que sean adecuadamente remunerados y apoyados en su trabajo.

Para ello, el informe determinó que existían varias dimensiones que afectan el pleno desarrollo profesional de los docentes: La formación inicial, el perfeccionamiento continuo, condiciones de trabajo en las escuelas y liceos y el ministerio que apoya eficientemente la profesión, el fin es favorecer el aprendizaje de los alumnos para que estos se puedan desenvolver en tres ámbitos: la inserción laboral, participación ciudadana y realización personal.

En términos generales, declaran que los/as profesores/as tienen grandes carencias y debilidades, propias de la etapa de transición que ha asimilado los cambios a nivel discursivo, y que no poseen las competencias para llevarlos a la práctica en el aula. (Beca y Otros: 2005).

\section{Asignación Variable por Desempeño Individual y Bonificación de Reconocimiento Profesional (2005)}

Dentro de los apoyos hacia la retención de docentes en las aulas, en 2005 se agrega otro hito, la Asignación Variable por Desempeño 
Individual, que corresponde a un estímulo económico dirigido a los/ as docentes del sector municipal que han obtenido buenos resultados en la Evaluación Docente.

Con posterioridad a las primeras iniciativas sobre Evaluación Docente, y las experiencias político-técnicas-educativas de la Comisión sobre Formación Inicial Docente (2005) y Docentes Para un Nuevo Siglo (2005), se realizan un conjunto de modificaciones y agregaciones a través de la Ley I9.997 (2005) y la Ley 20.I58, que establece diversos beneficios para profesionales de la educación y modifica distintos cuerpos legales, señalando la creación de "una Bonificación de Reconocimiento Profesional para los profesionales de la educación que se desempeñen en el sector municipal, particular subvencionado y en establecimientos de educación técnico-profesional regidos por el decreto ley $\mathrm{N}^{\circ} 3 \mathrm{I} 66$, de 1980, y que cumplan con los requisitos establecidos" (Ley 20.I58, 2006).

\section{Consejo Asesor Presidencial (2005)}

Otro interesante punto de inflexión en la trayectoria de la FID será originado producto de las movilizaciones estudiantiles durante el año 2006, también llamada 'Revolución Pingüina', que a juicio de Correa y Stahl dio cuenta de que la educación chilena, si bien exhibe bastantes logros, especialmente en cobertura, no alcanza resultados de aprendizaje satisfactorios, no muestra evidencia de mejoramiento sostenido y posee, en cambio, como una de sus características más notorias, un elevado nivel de segmentación y segregación social. (Bellei, et al., 2009).

Producto de meses de movilizaciones, la Presidenta de la República Michelle Bachelet, decide crear el Consejo Asesor Presidencial motivado por las movilizaciones de los jóvenes secundarios (Mineduc, 2006).

En cuanto a la FID, el Informe Final del Consejo realizó un crítico diagnóstico, estableciendo la existencia de: "falencias en la estructura, gestión y oferta curricular de la formación inicial; falta de regulación sobre las condiciones de apertura y funcionamiento de las carreras de Pedagogía; imprecisión acerca de las condiciones 
de funcionamiento de las carreras; expansión en los últimos años de 'programas especiales' de formación docente; las carreras de Pedagogía ofrecidas en la modalidad de 'programas especiales' no entregan a los futuros docentes oportunidades curriculares semejantes a las de los programas regulares; se mantiene una situación mixta en la formación docente: universitaria e institutos profesionales; la oferta de formación docente continua no siempre es apropiada para las necesidades de los profesores en servicio; inexistencia de canales institucionales, perfiles de competencias y programas definidos para la formación del profesorado de la educación media técnico-profesional; y en la educación especial tiende a prevalecer una formación basada en un enfoque clínico, centrada en el déficit, y no en un enfoque educativo centrado en la enseñanza y aprendizaje del currículo nacional, lo que dificulta la integración escolar de los alumnos con necesidades educativas especiales” (Mineduc, 2006: 174-I75).

El conjunto de nudos críticos planteados por el consejo señalaba la "ausencia de un sistema institucionalizado de regulación de la formación docente y de estímulo a su desarrollo y mejoramiento, y de medidas concretas que puedan disminuir el efecto de estos problemas”. (Mineduc, 2006: I75).

A su vez, en términos específicos se recomendaba a las instituciones formadoras: modificar los currículos de formación de modo de preparar profesores especialistas para los primeros seis años de la educación básica, ofreciendo formación en tres o cuatro áreas curriculares, hasta el $4^{\circ}$ grado; y en un máximo de dos, en los grados $5^{\circ}$ y $6^{\circ}$; vincular la formación de educadoras de párvulos y de profesores de enseñanza básica y estudiar ofrecer especialización en educación inicial (Kinder, $\mathrm{I}^{\circ}$ y $2^{\circ}$ años); establecer programas de formación (en conjunto con los departamentos especializados) para la educación media inferior, o grados $7^{\circ}$ a $\mathrm{IO}^{\circ}$ (en dos menciones), y para la educación media superior (en dos menciones a lo sumo, de áreas afines); preparar profesores especialistas en toda la secuencia escolar para las áreas curriculares de: Inglés, Educación Física, Artes Musicales y Artes Plásticas, con menciones en Pedagogía que distingan entre educación básica, concebida de acuerdo a la nueva estructura que propone este Informe (hasta grado 6), y media (grados 
$7^{\circ}$ a $12^{\circ}$ ); ofrecer programas de post-título que permitan especializar a profesores en servicio para alguna de las opciones señaladas en la recomendación. (Mineduc, 2006: 18I).

\section{Programa Inicia y Evaluación Docente}

Producto de lo anterior, y en referencia a experiencias internacionales (específicamente de EE.UU.) se da comienzo al Programa Inicia en 2007, como parte de una de las iniciativas consideradas en el programa FFID, el cual consistía en tres ámbitos: "la elaboración de estándares que buscan especificar y definir qué se espera de los egresados, qué se espera que sepan y sean capaces de hacer, como también orientaciones para la formación de profesores; definir un sistema de evaluación para dar cuenta del logro -o no logro- de dichos estándares, $y$, finalmente, el financiamiento de proyectos de mejoramiento para que las facultades de educación puedan alcanzar estos estándares en sus egresados” (Meckes, 2009).

Este conjunto de componentes se concretizan por el Ministerio de Educación a través del Centro de Perfeccionamiento, Experimentación e Investigaciones Pedagógicas (CPEIP), quien elaboró el programa, con el fin de fortalecer progresivamente la calidad de la formación profesional de los estudiantes de pedagogía a nivel nacional, fundamentado en las propuestas realizadas por diversas instancias de debate público, a saber: Comisión Nacional sobre Formación Inicial Docente (2005); Comisión Docentes para el Nuevo Siglo (2005) y el Consejo Asesor Presidencial de Educación (2006).

Respecto de la Evaluación Diagnóstica INICIA, se perfiló su importancia con el fin de otorgar insumos para que las instituciones formadoras mejoren su proceso formativo, siendo los resultados -en una instancia inicial- de carácter privado y confidencial.

De acuerdo con Manzi (2010: 286), el programa Inicia, en su dimensión de evaluación diagnóstica, "ha permitido (...) producir evidencia empírica consistente sobre algunas de las principales dificultades", acerca de los aspectos centrales de la FID: atracción, formación y retención de maestros/as. 
A la luz de lo expuesto anteriormente, es relevante enfatizar un conjunto de evidencias que la evaluación plantea: en primer lugar la alta participación en la prueba, que en su primera versión en 2008 congregó a un $80 \%$ del total de las instituciones formadoras, y la rendición efectiva de un $61 \%$ de los/as egresados/as inscritos por las instituciones. Otro antecedente interesante es el puntaje PSU de los evaluados/as, el cual es relativamente bajo, en tanto la mayoría se encuentra en un índice inferior a los 500 puntos, lo que se condice a su vez con la composición socioeconómica de los/as egresados/as, que en su mayoría provienen de hogares menos privilegiados, pues sólo un I3\% de ellos/as tienen padres con estudios universitarios completos, y un 49,4\% proviene de la educación municipalizada, tendencia que es específica en el área de estudios de educación.

En cuanto a los resultados de las diferentes pruebas, en el caso de la prueba de conocimientos disciplinarios sólo un $47 \%$ de los/as egresados/as obtuvieron respuestas correctas, lo cual correlaciona de forma significativa con los puntajes de ingreso, es decir mientras más bajo el puntaje PSU de ingreso a la carrera de pedagogía, menos cantidad de respuestas correctas obtienen los/as egresados/as. (Manzi, 2009; Meckes, 2009).

La evaluación diagnóstica Inicia y sus resultados, por una parte han ido entregando un conjunto de insumos claves para definir políticas hacia mejorar la FID, sin embargo, por otra parte ejercen una demanda/presión hacia las instituciones formadoras, que impulsada por la publicación de los resultados y el efecto ranking de 2010, tienden a ejercer un efecto modelador y estandarizado de la FID, imponiendo un discurso unívoco de la calidad en la formación de maestros/as. Esto último me parece peligroso y crítico en el contexto de una oferta de programas desregulada y poco relacionada con las necesidades del mercado laboral.

Asimismo, es preocupante dar cuenta acerca de la correlación existente entre los puntajes de ingreso y los resultados obtenidos en el test, por cuanto pone en jaque el valor agregado que la institución formadora tenga en las competencias del/la estudiante. 
En la misma línea de lo anterior, los resultados de la Evaluación Docente señalan para 2010 que de un total de II.063 docentes evaluados/as, un $69,4 \%$ corresponde a mujeres y un $30,6 \%$ a hombres, quienes se concentran mayoritariamente en los segmentos etarios de 40 a 49 años (22,7\%) y 50 a 59 (36,7\%), siendo en su mayoría de Enseñanza Básica (77,4\%).

En cuanto a sus resultados, el CPEIP señala que un 35,9\% de los docentes evaluados obtiene niveles de desempeño que no son adecuados, ya que un $6 \%$ se califica como destacado, un 58,1\% competente, un 33,3\% básico y finalmente un 2,6\% insatisfactorio. (MINEDUC, 20I0).

El efecto tanto de Inicia, como de la Evaluación Docente, han generado intensos debates acerca de las responsabilidades que le competen a la FID, y por ende a las instituciones formadoras. Ambas pruebas han publicado por la prensa los resultados, destacando los básicos resultados obtenidos, haciendo emerger una serie de supuestos, dentro de los cuales el más importante ha sido asociar la calidad de la educación como sistema, a la baja calidad de los/ as docentes.

Un elemento importante a considerar, es la promulgación de la Ley 20.501 en 20II, que establece que los equipos de gestión de los establecimientos educacionales "podrá(n) proponer anualmente al sostenedor el término de la relación laboral de hasta un 5\% de los docentes del respectivo establecimiento, siempre que hubieren resultado mal evaluados según lo establecido en el artículo 70 de esta ley; proponer al sostenedor el personal a contrata y de reemplazo, tanto docente como regido por la ley $\mathrm{N}^{\circ}$ 19.464; designar y remover a quienes ejerzan los cargos de Subdirector, Inspector General y Jefe Técnico del establecimiento de acuerdo a lo establecido en el artículo $34 \mathrm{C}$ de esta ley; ser consultado en la selección de los profesores cuando vayan a ser destinados a ese establecimiento; proponer al sostenedor los incrementos de las asignaciones contempladas en el inciso primero del artículo 47 y las asignaciones especiales de acuerdo a lo establecido en el inciso segundo del mismo artículo" (Ley 20.50I, 20II). 
Con ello, la evaluación docente se articula como una herramienta de control de la calidad, asociada a beneficios y sanciones, amparadas por ley, legitimando un modelo de calidad docente basado en las experiencias previas del proyecto FFID, las orientaciones de los Estándares de Desempeño 2000 y el Marco para la Buena Enseñanza 2003.

\section{Acreditación Obligatoria de los Programas de Pedagogía (2009)}

En cuanto a los recientes procesos de acreditación de las instituciones formadoras de docentes se constatan como debilidades: la escasa relación entre la especialidad y el área pedagógica en las carreras, el uso inadecuado de metodologías de enseñanza acorde con los objetivos de formación que se proponen, dificultades en las renovaciones de las plantas docentes. En términos de fortalezas se valora positivamente el uso de sistemas de prácticas profesionales tempranas y progresivas. Por otra parte, continúan existiendo nudos críticos en torno al grado de licenciado y la conveniencia de que las carreras universitarias de pedagogía tengan que otorgar el grado de licenciado antes del título de profesor. (Téllez, 2006).

Independiente de los indicadores de acreditación de las carreras de pedagogía, y la obligatoriedad por ley, resulta relevante contrastar los datos con las percepciones de los equipos directivos y formadores/as, en cuanto al grado de incidencia de los procesos de acreditación en las efectivas mejoras de los procesos formativos, en tanto las instituciones realizan una serie de medidas de mejora a raíz de la cantidad de años a obtener y el efecto de marketing asociado, más que como consecuencia de sus propios procesos internos de autoevaluación, por ende considero relevante formular políticas gubernamentales, pero también propias de las instituciones formadoras en relación a mejoras continuas, contextualizadas a sus necesidades específicas y sus posicionamientos y propuestas ante el sistema educativo en general, $y$ la FID en particular, más que como respuesta a las demandas externas. 


\section{Panel de Expertos para una educación de Calidad (2010)}

Otro hito fundamental, significó la realización del Panel de Expertos para una Educación de Calidad (2010), quienes elaboran el documento de "Propuestas para fortalecer la profesión docente en el sistema escolar chileno”. En dicho informe el Panel estima que es fundamental fortalecer las capacidades docentes del sistema escolar, considerando tres áreas prioritarias. (Mineduc, 2010c).

En lo específico de FID, el Panel señalaba la necesidad de "asegurar una buena formación inicial docente es una parte integral de la preocupación por allegar mejores capacidades al sistema escolar. Al respecto, ha constatado el fuerte aumento en los matriculados en las carreras de pedagogía (...) esa tendencia ha apartado al país de la voluntad expresa de seleccionar a sus futuros docentes de entre los jóvenes de mayores habilidades $[y]$ (...)estima que ha habido un retroceso en lugar de un avance, y cree urgente remediar esta situación, [en la medida que se produce] (...) una tensión evidente entre los objetivos declarados de lo que el país espera respecto de sus futuros profesores y su preparación, y la oferta actualmente disponible en el país” (Mineduc, 2010c).

Frente al diagnóstico realizado por esta instancia, el Panel estima que se deben incrementar las exigencias de calidad, las cuales deben ir de la mano de apoyos específicos a los programas que muestren un compromiso efectivo con una formación inicial docente de calidad. No obstante lo anterior, recomiendan en términos específicos acciones tendientes a: "fiscalizar que los programas que obtengan menos de cuatro años de acreditación no podrán obtener beneficios económicos del Estado para sus estudiantes; exigencia de aprobación de un examen de habilitación para poder enseñar en la educación subvencionada por el Estado; los desempeños de los egresados de los diversos programas de pedagogía deberán ser públicos; las instituciones formadoras tendrán que hacerse cargo obligatoriamente, a su costo, de la nivelación de sus egresados que, habiendo reprobado el examen de habilitación, aspiren a rendirlo por segunda vez; el examen estará a cargo de un Consejo que otorgue garantías de 
imparcialidad y calidad del instrumento; perfeccionar el sistema de acreditación de las carreras de pedagogía y que se incluya la obligación para estas de cumplir estándares exigentes y orientaciones curriculares básicas que estén en línea con las mejores prácticas internacionales en formación inicial docente". (Mineduc, 2010c: 59).

En síntesis, el panel de expertos reitera una serie de observaciones ya señaladas con anterioridad en el informe OCDE (2004), la Comisión Nacional de Formación Inicial Docente (2005), Docentes para un nuevo siglo (2005) y el Consejo Asesor Presidencial (2006), continuando una lógica política. No obstante, se delinean una serie de medidas explícitas y se enfatizan otras ligadas principalmente a políticas de atracción a la docencia y evaluación de los desempeños, sobre todo en cuanto a la realización de un examen de habilitación, que considere los aspectos mínimos que los/as docentes debiesen poseer para el ejercicio de la docencia. Respecto de ello, me parece que continúa la lógica de establecer un discurso homogéneo acerca de la calidad educativa, asociándola directamente a la calidad de las competencias de los/as profesores/as. No obstante, carece de una mirada sistémica, problematizada y contextual de la FID en nuestro país.

\section{Beca Vocación de Profesor (2011)}

Producto de las sugerencias del panel, en 20I I comenzó su puesta en práctica de la Beca Vocación de Profesor, consistente en el beneficio del pago del arancel a estudiantes que se matriculen por primera vez como alumnos/as de primer año en carreras de pedagogía elegibles y que se encuentren acreditadas, y para aquellos/as estudiantes que cursan último año de licenciatura no conducente a título profesional y que opten por el ciclo de formación pedagógica elegible para licenciados. Estos/as estudiantes deben haber obtenido al menos 600 puntos promedio PSU (Lenguaje y Comunicación y Matemáticas) al momento de ingreso a la carrera. (Mineduc, 20I Ib).

De acuerdo a la entrega del beneficio durante 201 I, la evidencia entregada por el DEMRE da cuenta del incremento en el porcentaje de alumnos seleccionados con más de 600 puntos en las carreras de 
pedagogía de las universidades del Consejo de Rectores (CRUCH), ya que en 2010 , un $26,8 \%$ del total de escogidos en dichas carreras obtuvo más de 600 puntos, mientras que en 20I I, esta cifra alcanzó un $34,2 \%$, con un aumento promedio de 57I,3 a 580,82, es decir 9,5 puntos. (Mineduc, 201 Ib).

Aun cuando la diferencia en el incremento de puntaje promedio de ingreso es de 9,5, considero que no es posible determinar con exactitud el grado de incidencia de este beneficio en la calidad de la FID, sin embargo es relevante mencionar que representa una de las pocas políticas -si no la única- dirigida directamente a la atracción de mejores puntajes de ingreso a la docencia.

\section{Estándares Orientadores para Egresados de Carreras de Pedagogía en Educación Básica (2011)}

Finalmente el hito que cierra este itinerario de la FID en Chile, es la elaboración y reciente publicación de los Estándares Orientadores para Egresados de Carreras de Pedagogía en Educación Básica (20IIa). Hay que recordar que la definición de este instrumento responde a los lineamientos del proyecto FFID, las sugerencias de las distintas instancias sobre formación docente anteriores, y específicamente como parte del programa Inicia, que consideraba dentro de su diseño la elaboración de estándares para profesores/ as egresados/as que enseñaran de $\mathrm{I}^{\circ}$ a $6^{\circ}$ básico. Este trabajo fue encargado en 2008 con el fin de "servir de orientación a las instituciones formadoras de docentes respecto a aquellos conocimientos y habilidades fundamentales para ejercer un efectivo proceso de enseñanza, respetando la diversidad existente de perfiles, requisitos, mallas curriculares, trayectorias formativas y sello propio, que caracterizan a cada una de dichas instituciones (Mineduc, 20I Ia).

La confección de los estándares, se encargó a dos instituciones: CIAE y CEPPE, pertenecientes respectivamente a la Universidad de Chile, a cargo de los estándares pedagógicos y disciplinarios de Lenguaje y Matemática, y la Pontificia Universidad Católica de Chile en Ciencias Naturales y Sociales. 
En términos del impacto de estos documentos, cabe señalar que declaran tener una doble función: un "qué", referido a un conjunto de aspectos o dimensiones que se debieran observar en el desempeño de un futuro profesor o profesora; y también, establecen un "cuánto" o medida, que permite evaluar qué tan lejos o cerca se encuentra un nuevo profesor o profesora de alcanzar un determinado desempeño, esperando que los estándares resultarán de utilidad para: "Tener visión de conjunto sobre conocimientos y habilidades profesionales, como, también, sobre el compromiso moral propios del profesor y profesora de Educación Básica; disponer de una referencia sobre lo que se espera de ellos al finalizar sus estudios; comparar, a lo largo del proceso de su formación, lo que han logrado respecto a una referencia”" (Mineduc, 201 Ia: 5).

En cuanto a la estructura de los Estándares, de acuerdo a las diferentes áreas de Lenguaje y Comunicación; Matemática; Historia, Geografía y Ciencias Sociales; y Ciencias Naturales, estos se han organizado en torno a dos grandes categorías: estándares pedagógicos y estándares disciplinarios, las cuales se articulan y complementan entre sí con el fin de proporcionar al futuro profesor los conocimientos y habilidades necesarios para el desempeño de la docencia.

En términos epistemológicos, los estándares se alinean a las propuestas de Competencias Genéricas de Tuning Europa-América Latina (2007), en tanto se decidió incorporar en los estándares aquellas características básicas que se espera logren los futuros/as profesionales chilenos/as. A su vez, se declara que el documento está enfocado para que los/as egresados/as enfrenten un entorno cambiante, a través del desarrollo de habilidades y actitudes personales tales como capacidad de trabajo colaborativo, autonomía, flexibilidad, capacidad de innovar, disposición al cambio y pro-actividad, y otras habilidades y disposiciones específicas, en coherencia con los aspectos evaluados en Inicia, considerando que a partir de 201 I, la evaluación diagnóstica se realiza -en el caso de Pedagogía en Educación Básicaen referencia a los Estándares (Mineduc, 20I I; Meckes, 2009). 


\section{Síntesis y desafíos actuales a la FID}

Este último hito, forma parte de una misma línea de políticas, delineadas desde el periodo I997-2002, tras el proyecto FFID, y recoge las orientaciones dadas por las comisiones de 2005, 2006 y 20I0, en conjunto con los resultados de la Evaluación Docente, AEP y preliminares de INICIA. Sin embargo también responden a políticas internacionales, que ya se han encaminado en la misma dirección. A su vez, desde una panorámica rápida no es sorprendente visualizar procesos similares en distintos países de la región, donde la fijación de estándares se asocia directamente con el establecimiento de un piso mínimo de calidad, que también se asocia a presiones directas e indirectas hacia las instituciones formadoras, en términos de sus rediseños curriculares y la asignación de recursos para los distintos proyectos de mejoramiento.

En este sentido, se está en presencia de una política sistemática de estandarización/homologación e intento de regulación de la FID, asociada a la atracción de los/as mejores (en términos de puntaje de ingreso PSU), demarcando la calidad de la formación (mediante la fijación de Estándares), y el egreso (mediante prueba Inicia).

Con ello los espacios formativos y propositivos de las instituciones formadoras se presentan limitados y establecidos con antelación, sin considerar elementos contextuales, modelos formativos específicos, asumiendo públicos objetivos homogéneos y a la calidad educativa y la FID unívoca y determinada.

Por otro lado, en un afán de sintetizar el itinerario de más de una década de políticas educativas en torno a FID en Chile, parece relevante dar cuenta de cómo ésta se articula en la actualidad. Al respecto Beatrice Ávalos (2008) da cuenta de la dinámica generada en los últimos años, donde el descenso de la demanda por ingresar a estas carreras observado desde 1985 comenzó a revertirse y aumentar de forma explosiva en todas las áreas ${ }^{2}$. Respecto de las trayectorias y

2 Educación Parvularia, Educación General Básica, Educación Diferencial y en algunas carreras de Educación Media (II \% en Educación Parvularia, 99\% en Educación Básica, 245\% en Educación Media y un 47\% en Educación diferencial). (CSE, 2008). 
programas, lo primero refiere a la duración en número de semestres, y lo segundo corresponde a programas según tipo: concurrentes que integran lo disciplinar y pedagógico, y lo consecutivo, donde primero se completa una formación disciplinar específica para luego generar la formación pedagógica.

El Informe Nacional del Estudio Internacional IEA TEDS-M (Ávalos \& Matus, 20I I) expone que la FID en nuestro país "no constituye un sistema propiamente tal, sino que un conjunto de instituciones y programas que han crecido en forma desreglada” (p. I35), no existiendo criterio de verificación de la calidad, con excepción de la ley 20.129 de acreditación obligatoria de carreras de pedagogía.

Respecto de las características de los/as estudiantes de pedagogía, "en general, los futuros profesores provienen de sectores socioeconómicos medio y medio-bajo, lo que es corroborado a partir del nivel educacional de los padres, en la amplia muestra de futuros profesores" (Ávalos, 2009: 263-264).

En cuanto a criterios de calidad y efectividad de la FID en Chile, según Ávalos (2009) y Manzi (2009), no existe suficiente evidencia para establecer relaciones entre calidad y efectividad, y se ha operado en relación a supuestos, tales como la relación entre la calidad de la educación recibida en las escuelas, en concordancia con los puntajes SIMCE, PSU, TIMMS y PISA, y la calidad de la formación recibida por los/as profesores/as en ejercicio.

Si bien las investigaciones internacionales señalan la relación existente entre la calidad de la docencia y la mejora de la calidad de la educación y los aprendizajes en las escuelas (OECD, 2004; Barber\&Mourshed, 2008), en nuestro país, de acuerdo a los/as autores/as, no se cuenta con evidencia empírica consistente al respecto, que permitan medir el impacto de la serie de políticas llevadas a cabo en los últimos años desde el proyecto FFID.

No obstante lo anterior, es fundamental relevar la importancia que factores externos poseen en la calidad de la FID, en tanto Mercado (2010) señala que la formación de maestros/as debe considerar un conjunto de aspectos relevantes, como sus experiencias previas, 
familiares, contextuales, percepciones, entre otras, que forman parte de un conjunto de discursos que lo constituyen, entendido como una forma 'dialógica' de concebir la formación (siguiendo a Bajtín).

Asimismo, Ávalos (2009a) señala que un factor de importancia que afecta a la FID, es el bajo nivel (puntaje PSU de ingreso) de quienes ingresan a las carreras de pedagogía, lo cual expresa las diferencias estructurales propias de la configuración socio-económica de Chile y las profundas brechas de igualdad de oportunidades, que se materializan en desiguales capitales culturales para afrontar el proceso formativo.

Finalmente, a partir de los diferentes análisis de la configuración actual de la FID en Chile, queda pendiente: trabajar en la calidad de los/as formadores/as de formadores/as, el tipo de oportunidades curriculares ofrecidas, las estrategias y las demandas de aprendizaje docente, la relación entre la evaluación de logros y estándares y competencias docentes.

Sin embargo, resulta pertinente y urgente revisar los discursos en torno a cómo se están concibiendo los procesos de diseño curricular de la FID hoy, a la luz de las innumerables exigencias y marcos regulatorios, que en la actualidad muestran un 'mínimo aceptable', pero también un 'máximo permitido' (Cherryholmes, I999), a través de los estándares y las evaluaciones al egreso.

La evidencia de esta investigación, señala que predominan discursos en el currículum de las instituciones formadoras, que se orientan a una 'formación integral', que se concibe como el conjunto/ sumatoria de todos los aspectos que idealmente debiese tener la formación inicial docente. Este discurso, se perpetúa y refuerza con la 'formación por competencias', modelo curricular predominante en la educación superior en Chile y el mundo. Cabe señalar que esta forma de concebir la formación docente proviene de una dinámica global de cambio en las últimas décadas, que apunta paulatinamente hacia una homologación de los sistemas a fin de generar los espacios de transferencia, flexibilidad y estandarización, propios del sistema económico predominante. 
Por otra parte, existen otros discursos que actúan en relación con los anteriores, y se expresan en términos de asociaciones causales, es decir, la relación entre la 'formación docente - calidad educativa', donde existe la creencia de que una formación de calidad de tipo disciplinar, redunda en la calidad de aprendizajes de quienes serán formados/as por los/as futuros/as docentes. Ahora bien, el discurso de la calidad no está claro, sin embargo es reiterativo y frecuente, haciéndole ver como uno solo, unívoco e incuestionado.

Asimismo, otra asociación causal discursiva corresponde a la 'formación docente de calidad y desarrollo del país', lo cual forma parte de otro discurso de carácter universal que refuerza la noción de que es a través de la educación que se producirá el desarrollo. De esta forma, aparecen otros conceptos aparentemente neutrales que se suman al de calidad, como el de 'desarrollo'.

Este conjunto de discursos, aparentemente objetivos y relativamente verdaderos, refuerzan y constituyen un 'orden del discurso' (Foucault, 1970), que genera una regulación social en la producción, recepción y circulación de los discursos (Popkewitz, 1994) sobre la formación inicial docente, que apuntan en el presente a una estandarización de la formación, promovido por las políticas públicas de este campo insertas en un mundo global que demanda cambios en esta ruta y no otras.

Este marco de acción limita la capacidad creadora y propositiva de las instituciones formadoras, y las políticas públicas instalan un proceso regulatorio cada vez más completo y complejo en los ámbitos de atracción, formación y retención, haciendo que la institución que forma docentes, aparezca 'respondiendo a la demanda', y no 'proponiendo ante la tensión', convirtiéndose en un interlocutor más de un discurso externo de informes e investigaciones sobre un concepto de 'calidad' aparentemente claro y resuelto, como objeto discursivo unívoco. 


\section{Referencias bibliográficas}

Ávalos, B. (2003). Profesores para Chile: Historia de un proyecto. Santiago: Ministerio de Educación.

Ávalos, B. (2008). Formación inicial de profesores. Formación inicial docente en Chile: calidad y políticas. En C. Bellei, D. Contreras \& J. Valenzuela (Edits.), Ecos de la Revolución Pinguina: Avances, debates y silencios de la reforma educacional. Chile: Universidad de Chile.

Ávalos, B. (2009b). La inserción profesional de los docentes. En Revista Profesorado: Revista de currículum y formación del profesorado. Vol. I3. NoI. 43 - 59. Recuperado el 20 de enero 2012, en www.redalyc. uaemex.mx/src/inicio/artpdffred.jsp?icue $=5671733004$

Ávalos, B. \& Matus, C. (20II). La Formación Docente en Chile desde una Óptica Internacional. Santiago: Ministerio de Educación.

Barber, M. \& Mourshed, M. (2008). Cómo hicieron los sistemas educativos con mejor desempeño del mundo para alcanzar sus objetivos. McKinsey \& Company. PREAL. Recuperado el día 20 de abril de 2012 en http:// www.oei.es/pdfs/documento_preal4I.pdf

Beca, C.; Montt, P. \& Sotomayor, C. (2006). Docentes para un nuevo siglo. Santiago: Mineduc.

Bellei, C.; Contreras, D. \& Valenzuela, J. (2009). Ecos de la Revolución Pinguina: avances, debates y silencios de la reforma educacional. Santiago: Universidad de Chile.

Cardelli, J. \& Duhalde, M. (200I). Formación docente en América Latina. Una perspectiva político-pedagógica. Cuadernos de Pedagogía (308).

Cherryholmes, C. (I999). Poder y Crítica: Investigaciones posestructuralistas en educación. Barcelona: Pomares Corredor.

Comisión Nacional de Acreditación (2002). Criterios de Acreditación de las Carreras de Pedagogía. Chile. Autor.

Comisión Nacional sobre Formación Inicial Docente (2005). Informe Final. Chile: Autor.

Comisión Nacional de Acreditación (2008). Acuerdos de Acreditación. Chile: Autor.

Comisión Nacional de Acreditación (2010). Informe de Resultados: CNA.

Consejo Asesor Presidencial para la Calidad de la Educación. (2006). Informe Final de Consejo Asesor Presidencial para la Calidad de la Educación. Santiago. 
Docentes para un Nuevo Siglo (2005). Informe Final. Chile: Autor.

Eurydice (2002, 2006). Formación inicial del profesorado y transición a la vida laboral. En Eurydice, The Teaching profession in Europe: profile, trends and concerns. General LOWER SECONDARY EDUCATION. Brussels: European Commmission.

Foucault, M. (1970). El orden del discurso. Lección inaugural en el Collège de France, pronunciada el 2 de diciembre de 1970.

IIPE-UNESCO (200I). Formación docente inicial. Informes Periodísticos. Buenos Aires: IIPE-UNESCO.

Informe Final del Proyecto Tuning América Latina: Reflexiones y perspectivas de la Educación Superior en América Latina (2007).

Latorre, M. (2005). Continuidades y rupturas entre Formación Inicial y Ejercicio Profesional Docente. Revista Digital OEI N०37.

Manzi, J. (2009). "Formación Inicial Docente: ¿Vamos por el Camino Correcto?". Documento presentado en el marco del seminario-taller. Santiago, Chile.

Manzi, J. (2010). Programa INICIA: fundamentos y primeros avances. En C. Bellei, D. Contreras, J. P. Valenzuela, Bellei, Contreras, C. Valenzuela, Daniel, \& Juan (Edits.), Ecos de la Revolución Pinguina: Avances, debates y silencios de la reforma educacional. (págs. 285 - 308). Santiago: Universidad de Chile.

Meckes, L. (2009). Programa para el mejoramiento de la formación inicial de profesores (INICIA). Santiago: MIDE-Universidad Católica y CEPPE Universidad Católica.

Mercado, R. (2010). El debate actual sobre la formación inicial de docentes en México. Revista Semestral da Associacao Brasileira de Psicología Escolar e Educacional., I4 (I), I49-I57.

Ministerio de Educación (2000). Estándares de Desempeño en la Formación Inicial de Docentes. Santiago: Mineduc.

Ministerio de Educación (2003). Marco para la Buena Enseñanza. Santiago: CPEIP.

Ministerio de Educación (2005). Informe Comisión sobre Formación Docente en Chile. Santiago, Chile: Mineduc.

Ministerio de Educación (2006). Consejo Asesor Presidencial. Informe Final. Chile: Autor.

Ministerio de Educación (2008). Resultados Evaluación Docente 2008: Mineduc. Recuperado el día 20 de marzo de 2012; en http://www. docentemas.cl/docs/ResultadosSIMCE2008.pdf 
Ministerio de Educación (2009a). Evaluación Diagnóstica Inicia. Presentación de Resultados 2009: Mineduc. Recuperado el día 20 de marzo de 2012 en http://www.evaluacioninicia.cl/docs/Inicia2009.pdf

Ministerio de Educación (2009b). Resultados Evaluación Docente 2009: Mineduc. Recuperado el día 20 de marzo de 2012, en http:// encuentros.universia.net/imagenesfotos/images_material_dc/5334I6I220-2010729-I0092I_I.pdf

Ministerio de Educación(2010a). Resultados Evaluación Docente 2010: Mineduc. Recuperado el día 20 de marzo de 2012 en http:/ / www.docentemas. cl/docs/20II/Resultados_Ev_Docente_2010_290320II.pdf

Ministerio de Educación (20I0b). Resultados Prueba Inicia Egresados Pedagogía en Educación Básica 2010: Mineduc. Recuperado el día 20 de marzo de 2012 en http://www.evaluacioninicia.cl/docs/Inicia2010.pdf.

Ministerio de Educación (2010c). Panel de Expertos para una Educación de Calidad. Chile: Autor.

Ministerio de Educación (201 Ia). Estándares Orientadores para egresados de carreras de Pedagogía en Educación Básica. Santiago: Mineduc.

Ministerio de educación (20I Ib). Resultados beca vocación de profesor 20I I: Mineduc.

OCDE (2004). Revisión de políticas nacionales de educación. Santiago, Chile: Autor. Recuperado el día 24 de abril, en HYPERLINK "http://www.oecd. org" www.oecd.org.

Panel de Expertos para una Educación de Calidad (2010). Propuestas para fortalecer la formación docente en el sistema escolar cbileno. Santiago.

Perrenoud, P. (200I). La formación de docentes en el siglo XXI. Revista de Tecnología Educativa, 3, 503-523.

Popkewitz, T. (I994). Política, conocimiento y poder: algunas cuestiones para el estudio de las reformas educativas. En Revista de Educación, núm. 305. Recuperado el día 25 de junio de 20I0, en HYPERLINK "http:/ / www. oei.es/reformaseducativas/politica_conocimiento_poder_popkewitz. pdf' http://www.oei.es/reformaseducativas/politica_conocimiento_ poder_popkewitz.pdf

Tellez, F. (2006). Caracterización de la formación inicial de las carreras de Pedagogía de las universidades chilenas que ban participado en el proceso de acreditación de la Comisión Nacional de Acreditación de Pregrado. Santiago: CPEIP.

Torres, R. (I996). ¿Nuevo rol docente?: Qué modelo de formación, para qué modelo educativo. Recuperado el 2I de noviembre de 20I I, de Fronesis: www.fronesis.org 
Vaillant, D. (2004). Construcción de la profesión docente en América Latina. Tendencias, temas y debates. Serie Documentos PREAL (3I), I-40.

Vaillant, D. (2009). Políticas para un Desarrollo Profesional Docente Efectivo. En D. Vaillant \& C. V. Medrano, Aprendizaje y Desarrollo Profesional Docente (págs. 29-39). Madrid: Santillana.

Villegas-Reimers (2002). La Formación Docente Inicial en los Estados Unidos de Norteamérica: tendencias recientes en sus prácticas políticas. En UNESCO, Formación docente: un aporte a la discusión. La experiencia de algunos paises. 